\title{
Handling Uncertainty and Finding Robust Pareto Frontier in Multiobjective Optimization Using Fuzzy Set Theory
}

\author{
Tohid Erfani* and Sergei V.Utyuzhnikov ${ }^{\dagger}$ \\ The University of Manchester, School of Mechanical, Aerospace and Civil Engineering, \\ Manchester, M60 1QD, Great Britain
}

\begin{abstract}
Multiobjective optimization problem with uncertainties in the input data is considered. Due to the uncertainty, the use of deterministic approaches for finding the solution to the problem is problematic. Statistical techniques based on probability distributions are not efficient if the input data are not sufficient to estimate the parameters of the model accurately enough. An additional problem arises if the probability distribution for the available data is unknown. Meanwhile, the partial information available to the decision maker can be useful if fuzzy information is exploited. In this paper, we introduce the definition of optimality using fuzzy variables to handle the uncertainty in the model. Thereafter, we extend the concept of robust frontier to the model based on fuzzy information. We make it possible to find a less sensitive frontier in multiobjective optimization with partial information. The approach is illustrated by solving a test case, well-known in the literature.
\end{abstract}

\section{Nomenclature}

\author{
$\mathcal{D} \quad$ Design space \\ $\mathcal{F}$ Objective function vector \\ $\alpha \quad \alpha$-cut of fuzzy number \\ $\sigma \quad$ Variation in design variable/parameter \\ $\mathcal{R}_{\mathcal{F}} \quad$ Robustness measure \\ Sub- and Super-script \\ $\sim \quad$ Uncertain value \\ pm Possibilistic mean value \\ $n \quad$ Number of objective functions \\ $m$ Number of design variables/parameters
}

\section{Introduction and Background}

In the real-life multidisciplinary design there is an uncertainty in the input data. In this environment, the decision maker (DM) usually prefers to have a solution not much sensitive to small variation in model's parameters and variables. In this context there are two major tasks to be done. First, the uncertainty in model parameters should be handled and the model should be converted into the conventional optimization problem. Second, the solution, which is less sensitive to the input variations, should be found.

For the first task, many researches assume that the uncertain parameters are random, and adequate data are available to estimate the probability distribution of their variation. In such scenarios, probability theory and stochastic programming ${ }^{1}$ is used to obtain the solution. In stochastic techniques, the constraints are first

*Post Graduate Researcher, AIAA Student Member, Email: Tohid.Erfani@postgrad.manchester.ac.uk

†Senior Research Fellow, Email: S.Utyuzhnikov@manchester.ac.uk 
converted into the probabilistic constraints and then some techniques such as Monte-Carlo simulation are used to evaluate the probability of satisfaction (or failure) of each constraint. ${ }^{2}$ When the uncertainty does not fully behave randomness, uncertain variables defined by Liu in Ref. ${ }^{3}$ can be applicable. In the uncertain programming, some measure values (e.g. optimistic or pessimistic) for each constraint and objective function are formulated to substitute for the uncertain model. ${ }^{3}$ However, in many occasions, the uncertainties may come from imprecision and ambiguity of the parameters. In this instance, the problem can be handled using the fuzzy theory. For this purpose, the membership functions for each constraint and objective are constructed. Mujumdar et $a l^{4}$ use grey numbers rather than real ones to address the uncertainty in the model. They maximize the level of goal fulfillment by using the $\alpha$-cut of fuzzy parameters and generate a set of optimal interval-valued solution instead of a single set. Dubois and Prade ${ }^{5}$ introduce four inequality indices between fuzzy numbers and propose the treatments of linear programming problems with fuzzy coefficients. Each index is selected to satisfy the required assurance of DM. Inuiguchi ${ }^{6}$ uses the concept of modality in linear multiobjective optimization based on possibility and necessity measures. He transforms the model, using the DM level of uncertainty, into the conventional linear programming problem. The goodness of a fuzzy number instead of a random variable is that it does not require huge source of data and can be applicable with less and partial information from the DM. It can be carried out with ambiguous coefficients as well as vague preferences.

The purpose of the second task is to find the less sensitive or so called robust solution which is of high importance from practical standpoint. Parkinson et $a l^{7}$, use the analysis of tolerances to show how tolerances in design variables and parameters are transmitted into the constraints and objective functions. $\mathrm{Su}$ and Renaud ${ }^{8}$ introduce sensitivity-based and experimental-based robust optimization in single objective optimization to find the less sensitive optimum solution. The essence of their method is to study the vicinity of current design and hence find the solution which is less sensitive to fluctuations of parameters and design variables. In the multiobjective optimization, on the other hand, Deb and Gupta, extend an approach to optimize the mean effective objective functions. ${ }^{9}$ They introduce two types of robust solution and consider four probable cases which can happen in the real-life design. However, as argued in Ref., ${ }^{9}$ depending on the number of sample points, this method can be fifty times computationally expensive as much as regular optimization methods. In the multiobjective optimization, also, Messac and Yahaya ${ }^{10}$ develop a flexible physical-programming based robust optimization method to find a system with a minimal variation in the input variability and uncertainties. They consider robust design with both prescribed and variable tolerances, in which the variable tolerance level is determined optimally in the design phase. Like in Ref., ${ }^{7}$ they use the Taylor series in vicinity of each design to study the variation and find a less sensitive solution.

In the current paper we use the fuzzy numbers to show the existing uncertainty of the model. For the first task we define the notion of Possibilistic Mean optimality, and by using it, we defuzzify the model into the deterministic one. Thereafter, to resolve the second task, we develop the measure of robustness introduced in Ref. ${ }^{11}$ to multiobjective optimization. Then, using the variance of fuzzy numbers, we extend the analysis of tolerances for the fuzzy functions to find a more robust solution.

\section{Fuzzy multiobjective optimization}

The notion of fuzzy in the multiobjective optimization problems implies that the model is due to uncertainty. The DM does not know the parameters exactly and imprecision and ambiguity exist in the model used. A typical fuzzy multiobjective optimization problem can be formulated as follows:

$$
\begin{aligned}
\text { Min } & \tilde{\mathcal{F}}=\left\{\tilde{F}_{1}(x), \tilde{F}_{2}(x), \ldots, \tilde{F}_{n}(x)\right\}, \\
\text { subject to } & \widetilde{g_{i}(x)} \leq \tilde{b}_{i} \quad i=1 \ldots m, \\
& x \in \mathcal{D},
\end{aligned}
$$

where tilde means the functions contain fuzzy parameters.

To solve the problem defined in Eq. 1, we introduce the following definition. ${ }^{12}$ This definition can be used to substitute the parameters of the problem for their fuzzy mean value. The fuzzy mean value then can be used as the nominal value of the parameters. 
Definition 1 (Possibilistic Mean value of fuzzy number) If $A$ is a fuzzy number, its possibilistic mean value is the arithmetic mean of its lower and upper possibilistic mean value, i.e.

$$
\operatorname{Mean}(A)=\frac{M(A)+\bar{M}(A)}{2},
$$

where

$$
\underline{M}(A)=2 \int_{0}^{1} \alpha \underline{A} d \alpha \quad, \bar{M}(A)=2 \int_{0}^{1} \alpha \bar{A} d \alpha
$$

and $\bar{A}$ and $\underline{A}$ are the lower and upper bounds of $\alpha$-cut (Look at Appendix A) of fuzzy number $A$, respectively.

The problem in Eq. 1 is then reduced to

$$
\begin{aligned}
\text { Min } & \mathcal{F}_{p m}=\left\{F_{1}^{p m}(x), F_{2}^{p m}(x), \ldots, F_{n}^{p m}(x)\right\} \\
\text { subject to } & g_{i}^{p m}(x) \leq b_{i}^{p m} \quad i=1 \ldots m \\
& x \in \mathcal{D}
\end{aligned}
$$

where the $p m$ notation of problem implies that the fuzzy possibilistic mean value of parameters have been used.

In the problem defined in Eq. 2, we look for a set of solutions called Possibilistic Mean Pareto according to the following definition:

Definition 2 (Possibilistic Mean (PM) Pareto Optimality) Vector $x^{*}$ is called Global Possibilistic Mean Pareto solution to the multiobjective optimization of the form of Eq. 2 iff $\exists x^{* *}$ such that, $F_{i}^{p m}(x)^{* *} \leq F_{i}^{p m}\left(x^{*}\right)$ for any $i=1, \ldots, n$ and exists $j(1 \leq j \leq n): F_{j}^{p m}(x)^{* *}<F_{j}^{p m}\left(x^{*}\right)$. The $x^{*}$ is Locally Possibilistic Mean Pareto solution iff the above condition holds in $\epsilon$-vicinity of $x^{*} . x^{*}$ is the solution which is not dominated by any other feasible solution.

\section{Searching for robust solution}

We are looking for a robust solution, which means that it is less sensitive to the variation in parameters and design variables of the problem 2. For this purpose, we shift the solution into the feasible space. This will guarantee that the design will remain feasible even with small perturbation of the parameters. To realize this, we need the transmitted variation in variables/parameters into the constraints. Using the Taylor series as in Ref., ${ }^{7}$ the variation of a function transmitted into it is calculated by

$$
\sigma_{z}^{2}=\sum_{i=1}^{n}\left(\frac{\partial z}{\partial x_{i}}\right)^{2} \sigma_{x_{i}}^{2}
$$

where $\sigma_{x_{i}}$ is the variation in variables/parameters.

Therefore, for the constraint defined as

$$
g_{i}(x) \leq b_{i}
$$

the total variation is

$$
\sigma_{i}^{2}=\sigma_{g_{i}}^{2}+\sigma_{b_{i}}^{2},
$$

where $\sigma_{b_{i}}$ is the variation in the right-hand side of the constraint and $\sigma_{g_{i}}$ is calculated using Eq. 3. For the sake of using fuzzy numbers, the variation of the parameters and the right-hand side of the constraints, should be replaced by the variance of fuzzy parameters based on the following definition: ${ }^{12}$ 
Definition 3 (Variance of fuzzy number) If $A$ is a fuzzy number, the variance of it is defined as

$$
\operatorname{Var}(A)=\frac{1}{2} \int_{0}^{1} \alpha(\bar{A}-\underline{A})^{2} d \alpha .
$$

Having applied the transmitted variation for each constraint, we would have the multiobjective optimization problem with the form

$$
\begin{aligned}
\text { Min } & \mathcal{F}_{p m}=\left\{F_{1}^{p m}(x), F_{2}^{p m}(x), \ldots, F_{n}^{p m}(x)\right\}, \\
\text { subject to } & g_{i}^{p m}(x) \leq b_{i}^{p m}-\sigma_{i} i=1 \ldots m, \\
& x \in \mathcal{D}
\end{aligned}
$$

where $\sigma_{i}$ is to be found using Eq. 4. In the above formulation (Eq. 5), we subtract the total variation in constraint $i$ from the right-hand side. This shifts the solution point into the feasible space and keeps the feasibility of a design in presence of the variables/parameters perturbation. Moreover, to maximize the robustness, we define the measure of robustness as follows:

$$
\mathcal{R}_{\mathcal{F}}=\frac{1}{n m} \sum_{i=1}^{n} \sum_{j=1}^{m} \frac{\sigma F_{i}}{\sigma x_{j}},
$$

where $m$ and $n$ are the number of design variables/parameters and objective functions, respectively. Although the estimation of the dominator is straightforward (variance of the fuzzy number), calculation of the nominator requires efforts using Eq. 3.

Eq. 6 implies that the variation in design variables/parameters and the variation in objectives of the problem has an inverse relation. Therefore, it is clear that minimizing this value results in achieving a more robust solution. This is because of the fact that minimization of $\mathcal{R}_{\mathcal{F}}$ results in a design with less value of $\sigma F$ which leads to high value of $\sigma x$. This is exactly one may expect from a robust solution. Therefore, we add $\mathcal{R}_{\mathcal{F}}$ as a new objective function for Eq. 5. Hence, final formulation for searching the robust solution in multiobjective optimization might be given by:

$$
\begin{aligned}
\text { Min } & \mathcal{F}_{p m}=\left\{F_{1}^{p m}(x), F_{2}^{p m}(x), \ldots, F_{n}^{p m}(x)\right\}, \\
\text { Min } & \mathcal{R}_{\mathcal{F}}, \\
\text { subject to } & g_{i}^{p m}(x) \leq b_{i}^{p m}-\sigma_{i} \quad i=1 \ldots m \\
& x \in \mathcal{D} .
\end{aligned}
$$

Using the above formulation, the robust solution can be generated. It should be noted that, regardless of the number of objective functions in the original design problem, the new formulation will only have one extra objective function; the robust measure. The defined robust measure may be considered as a soft constraint from a different perspective, which needs to be minimized as much as possible.

\section{Test case: Two-bar truss structure}

In this section we consider an engineering test problem to investigate the introduced model for finding the robust design. A symmetric two-bar truss structure is a popular structural design which needs to be studied for the best, however robust, configuration. The test case is taken from Ref. ${ }^{10}$ and is shown in Figure 1. The structure is supposed to support a load $F$. The truss consists of two steel tubes pinned together at one end and supported on the ground at the other. The design variables are the diameter of members $\left(x_{1}\right)$ and the height $\left(x_{2}\right)$ of structure. We consider two objectives of the design: (i) minimize total mass of truss members and (ii) minimize the vertical deflection due to the application of the load $F=(150,20,30) \mathrm{kN}$ as triangular fuzzy number. As can be seen, these two objectives are in conflict. So multiobjective optimization is needed for finding a trade-off set of solutions. The parameters of the problem are the two fuzzy numbers: member thickness $t=(2.5,0.5,1.5) \mathrm{mm}$ and structure width $w=(750,100,50)$, and the two crisp (real) numbers: mass density $\rho=7.8 \times 10^{-3} \mathrm{gr} / \mathrm{mm}^{3}$ and Elastic modulus $E=210000 \mathrm{Nm}^{2}$. The normal stress has to be less than the buckling stress as constraint and $1 \leq x_{1} \leq 100$ and $10 \leq x_{2} \leq 1000$ must hold. Therefore, the formulation takes the following form: 

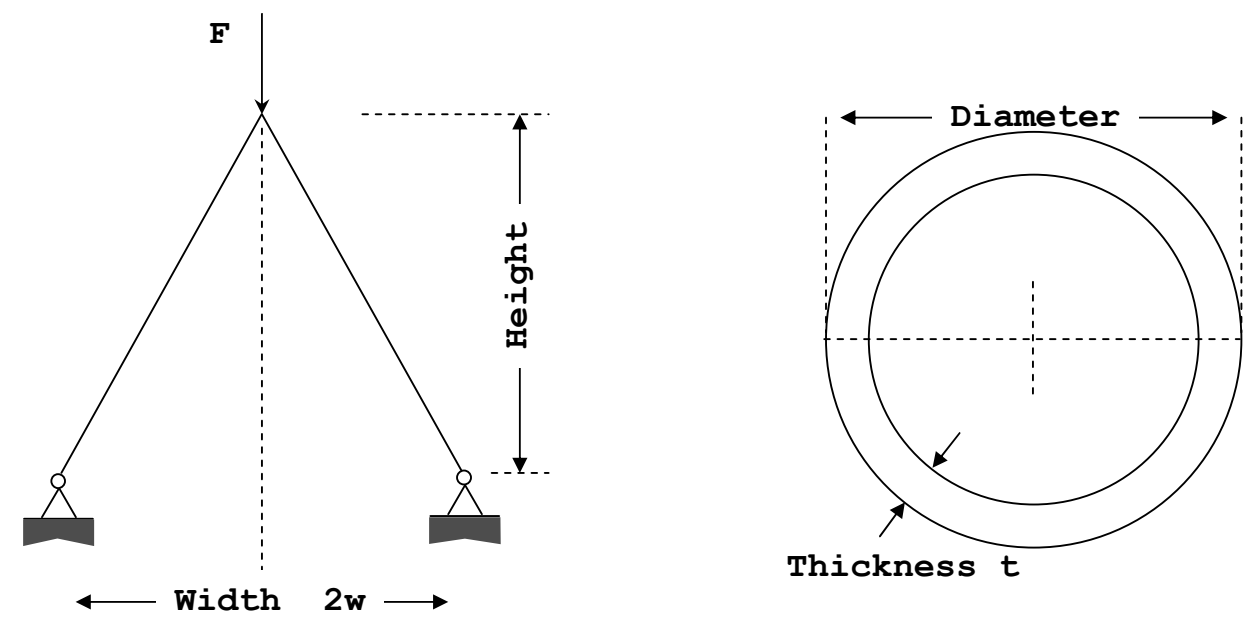

Figure 1. Two-bar truss. Structure (left), Section of member (right)

$$
\begin{aligned}
\text { Minimize } & \text { Mass }=2 \pi \rho t x_{1} \sqrt{w^{2}+x_{2}^{2}}, \\
\text { Minimize } & \text { Deflection }=\frac{F\left(w^{2} x_{2}^{2}\right)^{3 / 2}}{\left(2 \pi t E x_{1} x_{2}\right)^{2}}, \\
\text { s.t. } & s \leq \frac{1}{8} \pi^{2} E \frac{t^{2}+x_{1}^{2}}{w^{2}+x_{2}^{2}} \\
& 1 \leq x_{1} \leq 10 \\
& 100 \leq x_{2} \leq 1000
\end{aligned}
$$

where

$$
s=\frac{P}{2 \pi t x_{1} x_{2}} \sqrt{w^{2}+x_{2}^{2}}
$$

For this test case, we only consider the uncertainty in the parameters. The triangular fuzzy parameters are substituted by their possibilistic mean value of $F=151.6 \mathrm{kN}, t=2.66 \mathrm{~mm}$ and $w=741.6 \mathrm{~mm}$ (See Appendix A). The robust measure is then constructed with the variance of 1020.6, 0.4 and 30.61 for $F, t$ and $w$, respectively (See Appendix A). The constraints are modified and the problem of Eq. 7 is formulated. Using the DSD method ${ }^{13}$, the PM and robust Pareto frontiers are generated and are shown in Figure 2.

As can be seen, the robust frontier (white circles) is moved inside the feasible space with respect to the PM Pareto frontier. This is because of the shrinking of the feasible space under the new formulation for the sake of searching the robust frontier. This generates the solutions which are more reliable about the variation in design parameters. Using the DSD method, ${ }^{13}$ the robust frontier is generated and is shown by white circles. All these solutions are less sensitive to the parameters variations. However, looking at Figure 2 , given the enough resources and DM's situation, it is important for the design to be chosen with more value of mass and less value of deflection. This is because of the fact that the robust solution in this region (shown in Figure 2) deviates from the PM Pareto frontier less than the other design candidates. That is, not only are these solutions near optimal but also they are robust. It is worth noting that, even before optimization, it is intuitive to design these trusses with large mass in order to have the less deflection. This is equivalent to choose the solution from the indicated region (Figure 2) as it is found exactly by the optimization formulation. 


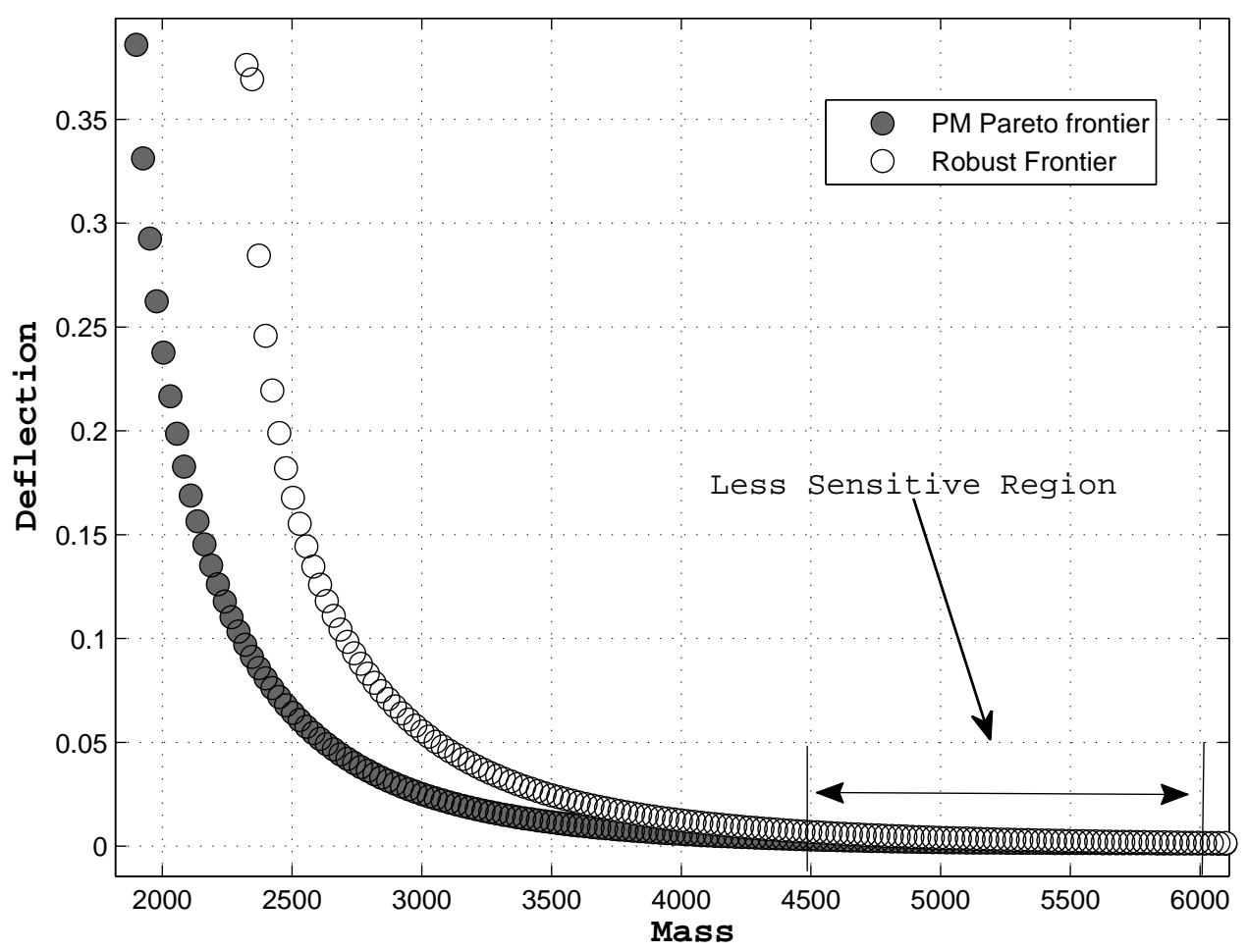

Figure 2. PM and Robust frontier for two-bar truss test case

\section{Conclusion}

In this paper we proposed an integrated approach in multiobjective design and optimization to deal with the uncertainty using the fuzzy set theory. The advantage of using the fuzzy number instead of random variable is that it gives the DM the flexibility to model with partial information where doubt about the exactness of a parameter values exists. Hence, the modeling can be done in more flexible way which is of use in practical application. The mean value of the fuzzy number has been used as the nominal value to handle the uncertainty in the model. Having implemented the analysis of tolerance, the variation in the constraint functions are calculated. To do this, the variance of fuzzy number as the variation in parameters has been exploited. To account for the minimal variations, a measure for the robustness has been proposed to incorporate the robustness concept into the problem. This function has been considered as a new objective function to the new formulation. The efficiency of the approach has been illustrated with a practical engineering example. As a future work it is important for the DM to be able to have control on the level of robustness for the design. This brings a good opportunity to study different kinds of robust frontiers and choose an appropriate design accordingly.

\section{Appendix A. Fuzzy numbers}

The $\alpha$-cut of a fuzzy number is defined as follows:

$\alpha$-cut of fuzzy set If $\tilde{A}$ is a fuzzy set, the crisp set of the elements belong to $\tilde{A}$ at least to the degree of $\alpha$ is called $\alpha$-cut of $\tilde{A}$ :

$$
\tilde{A_{\alpha}}=\left\{x \in X \mid \mu_{\tilde{A}}(x) \geq \alpha\right\},
$$

where $\mu_{\tilde{A}}(x)$ is the membership function of fuzzy number $A$. 
As an example, suppose $A$ is triangular fuzzy number shown by membership function $A=(a, b, c)$, where $b$ and $c$ are the left-width and right-width of the fuzzy number centered at $a$. Therefore, $\alpha$-cut of $A$ is computed by

$$
A_{\alpha}=[a-(1-\alpha) b, a+(1-\alpha) c], \quad \forall \alpha \in[0,1] .
$$

Furthermore, if $A$ is a triangular fuzzy number shown by $A=(a, b, c)$, it is easy to prove that the possibilistic mean value of the fuzzy number is given by

$$
M(A)=a+\frac{c-b}{6}
$$

and also its variance is computed as

$$
\operatorname{Var}(A)=\frac{(b+c)^{2}}{24}
$$

\section{Acknowledgments}

The first author gratefully acknowledges the $\mathrm{PhD}$ research scholarship awarded by the School of MACE, the University of Manchester. The valuable comments and remarks from unknown referees are appreciated.

\section{References}

${ }^{1}$ Kibzun, A. and Kan, Y., "Stochastic programming problems with probability and quantile functions," Journal of the Operational Research Society, Vol. 48, No. 8, 1997, pp. 849.

${ }^{2}$ Cruse, T., Reliability-based mechanical design, CRC Press, 1997.

${ }^{3}$ Liu, B., "Uncertainty theory: an introduction to its axiomatic foundations," 2004.

${ }^{4}$ Mujumdar, P. and Karmakar, S., "Grey Fuzzy Multi-Objective Optimization," .

${ }^{5}$ Dubois, D. and Prade, H., "Ranking fuzzy numbers in the setting of possibility theory," Information Sciences, Vol. 30, No. 3, 1983, pp. 183-224.

${ }^{6}$ Inuiguchi, M., "Multiple objective linear programming with fuzzy coefficients," Multiple Criteria Decision Analysis: State of the Art Surveys, Springer, New York, 2004, pp. 723-760.

${ }^{7}$ Parkinson, A., Sorensen, C., and Pourhassan, N., "A general approach for robust optimal design," Journal of Mechanical Design, Vol. 115, 1993, pp. 74 .

${ }^{8} \mathrm{Su}$, J. and Renaud, J., "Automatic differentiation in robust optimization," AIAA Journal-American Institute of Aeronautics and Astronautics, Vol. 35, No. 6, 1997, pp. 1072-1079.

${ }^{9}$ Deb, K. and Gupta, H., "Searching for robust Pareto-optimal solutions in multi-objective optimization," Proceedings of the Third Evolutionary Multi-Criteria Optimization (EMO-05) Conference (Also Lecture Notes on Computer Science 3410), Springer, 2005, pp. 150-164.

${ }^{10}$ Messac, A. and Ismail-Yahaya, A., "Multiobjective robust design using physical programming," Structural and Multidisciplinary Optimization, Vol. 23, No. 5, 2002, pp. 357-371.

${ }^{11}$ Jin, Y. and Sendhoff, B., "Trade-off between performance and robustness: An evolutionary multiobjective approach," Lecture notes in computer science, 2003, pp. 237-251.

${ }^{12}$ Carlsson, C. and Fuller, R., "On possibilistic mean value and variance of fuzzy numbers," Fuzzy sets and systems, Vol. 122, No. 2, 2001, pp. 315-326.

${ }^{13}$ Erfani, T. and Utyuzhnikov, S. V., "Directed Search Domain: A Method for Even Generation of Pareto Frontier in Multiobjective Optimisation," Engineering Optimization, To appear 2010. 